\title{
MEDICAL DOCTORS' ATTITUDES TOWARDS PRESCRIBING DRUG TREATMENT FOR BENIGN PROSTATIC HYPERPLASIA
}

\author{
Steliana Yordanova ${ }^{1}$, Galina Petrova ${ }^{2}$, Todorka Kostadinova ${ }^{2}$, Jivko Kolev ${ }^{3}$ \\ ${ }^{1}$ Master of Pharmaceutical management, Medical University of Varna \\ ${ }^{2}$ Department of Economics and Healthcare Management, Faculty of Public Health, \\ Medical University of Varna \\ ${ }^{3}$ Department of Pharmaceutical Sciences and Pharmaceutical Management, \\ Faculty of Pharmacy, Medical University of Varna
}

\begin{abstract}
INTRODUCTION: Benign prostatic hyperplasia is a disease that mainly affects men aged over $\mathbf{5 0}$ years. In Europe, cases of moderate and severe symptoms vary between 14 and 30\%. The therapy of BPH includes various methods and is intended to bring about an improvement in symptoms (irritative or obstructive), urodynamic function and quality of life.

The objective of this article is to present a survey into the attitudes of a group of urologists, providing care in outpatient and inpatient settings, towards therapeutic approaches in the treatment of benign prostatic hyperplasia (BPH) and the level of familiarity and preference for original Tamsulosin $0.4 \mathbf{~ m g}$.

MATERIALS AND METHODS: Sociological method: a structured interview with 73 urologists countrywide; Documentary method: research into regulatory documents; Statistical methods: for data processing and analysis of the collected information.
\end{abstract}

RESULTS: In the opinion of the interviewed urologists, original Tamsulosin is the first choice of medication for the management of $\mathrm{BPH}$.

Keywords: attitudes, doctors, treatment, $B P H$

Address for correspondence:

Galina Petrova

Faculty of Public Health

Department of Economics and Healthcare Management

Medical University of Varna

55 Marin Drinov Str.

9002 Varna, Bulgaria

e-mail:gal_rumenova@abv.bg

Received: March 1, 2015

Accepted: May 10, 2015

\section{INTRODUCTION}

$\mathrm{BPH}$ is a histological diagnosis characterised by hyperplasia of epithelial and stromal prostate cells; a non-malignant enlargement of the prostate gland which starts with the formation of small nodules in the transition zone of the prostate near the urethra. During its development the prostate gland tissue also proliferates and nodules become larger, which can affect the central and peripheral zones. The causes are still unclear but two main factors are indicated - ag- 
ing and androgens. In the process of aging the levels of testosterone decrease whereas those of estrogen increase, the so called andropause. It is considered that this increases the androgen sensitivity of the prostate tissue and directly causes hyperplasia of the stromal cells. The main hormone for the prostatic growth is the androgen dihydrotestosterone (DHT). It is synthesised from testosterone through the enzyme $5 a$-reductase and binds with the androgen receptors causing prostatic growth. In $\mathrm{BPH}$ patients both the enzyme and the receptor number is increased. The condition is not a problem in itself until some symptoms connected with the process start to manifest (1).

Tamsulosin $0.4 \mathrm{mg}$ is used for the treatment of $\mathrm{BPH}$. This is a selective alpha ${ }_{1 \mathrm{~A}}$-adrenoceptor blocking agent. It reduces the tension in the smooth muscles of the prostate and the urethra allowing urine to pass more easily through the urethra and facilitating urination. Moreover, it reduces the uncontrollable urge to urinate. The product is used by men in the treatment of lower urinary tract symptoms, associated with enlarged prostate gland (BPH). These symptoms may include difficulty urinating (weak stream), intermittent steam, urgency to urinate and urinary frequency both at night and during the day $(2,3)$.

Since its registration in Europe and USA, among urologists, Tamsulosin has become the most frequently prescribed alpha adrenergic receptor antagonist for the reduction of lower urinary tract symptoms and for improvement in the maximal flow rate in $\mathrm{BPH}$ patients due to the following factors $(2,3)$ :

1. No need for titration of the dose because alpha1 A selectivity prevents effects on the arterial blood pressure.

2. Fast relief of symptoms since the initial dose of $0.4 \mathrm{mg}$ daily is the therapeutic dose.

3. No need for change in the dosage of most frequently applied antihypertensive agents;

4. Reduced frequency of cardiovascular adverse effects (dizziness, asthenia, orthostatic hypotension) compared with generic products.

\section{RESULTS AND DISCUSSION}

The structured interview was carried out in June and July 2014. The target group of the survey were 73 urologists from the outpatients' and inpatients' services throughout the country which com- prises $21 \%$ of the urologists in Bulgaria. From the 73 interviewed urologists, $98.6 \%$ were men and 1.4\% women. Age distribution across the surveyed target group is the following: younger than 40 years of age $26 \%$; $41-50$ years $-30.14 \%$; $51-60$ years $-34.25 \%$; over 60 years $-9.59 \%$ (Fig. 1 ).

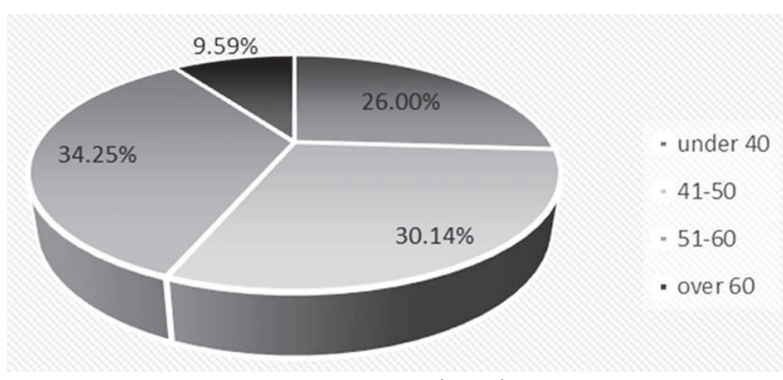

Fig. 1. Respondents' age

The majority of the respondents have less than 10 years of experience $-39.62 \%$, followed by between $11-20$ years - $37.88 \%$. 25.29\% have experience between 21-30 years and those between 31-40 years are just $7.22 \%$ (Fig. 2).

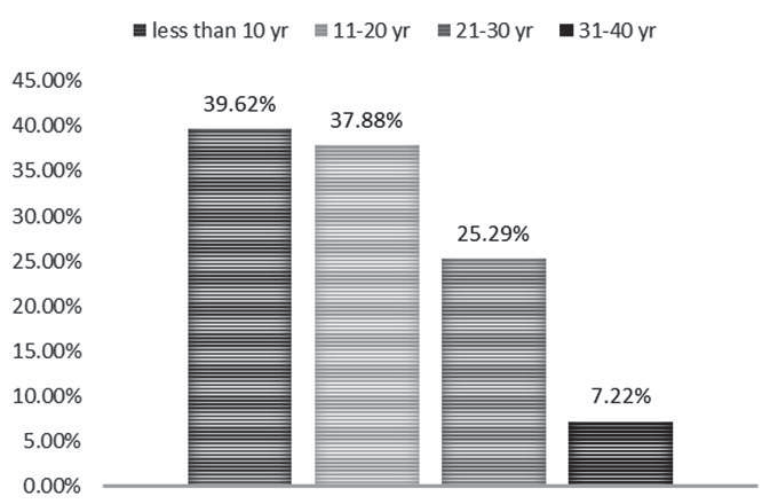

Fig. 2. Experience in the speciality

A matter of interest is the question defining the percentage of patients with $\mathrm{BPH}$ (What is the percentage of patients that you have diagnosed with Benign Prostatic Hyperplasia/BPH?).

Fig. 3 shows that in the practice of 49 out of all the urologists, the percentage of patients with $\mathrm{BPH}$ varies between $51 \%$ and $70 \%$; in the practice of 14 of the physicians, the percentage of patients with $\mathrm{BPH}$ is 31\%-50\%; 7 urologists report that their patients with $\mathrm{BPH}$ are over $70 \%$; and only 3 of the specialists report that the patients with this diseases are below 30\%. 
Steliana Yordanova, Galina Petrova, Todorka Kostadinova et al.

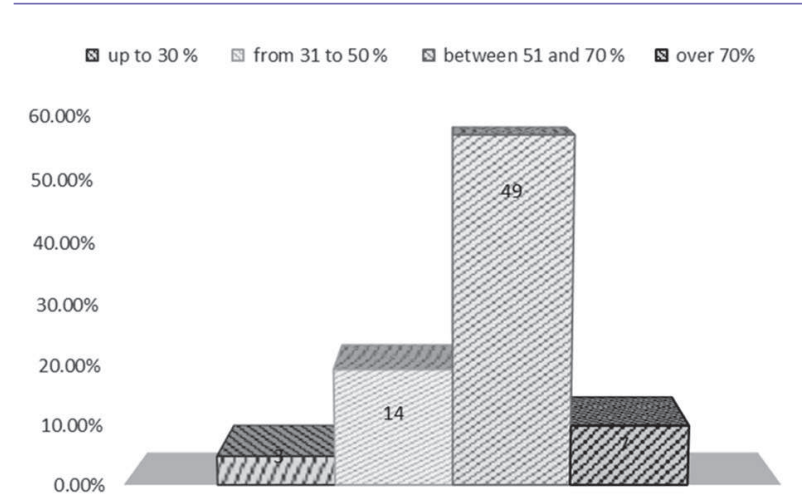

Fig. 3. Patients suffering from $B P H$

As evident from Fig. 4, 67.12\% of the respondents answered that more than half of their patients with lower urinary tract symptoms associated with $\mathrm{BPH}$ undergo tests and examination every 6 months. Only $30.14 \%$ of the doctors answered that less than half of their patients have periodical follow-ups.

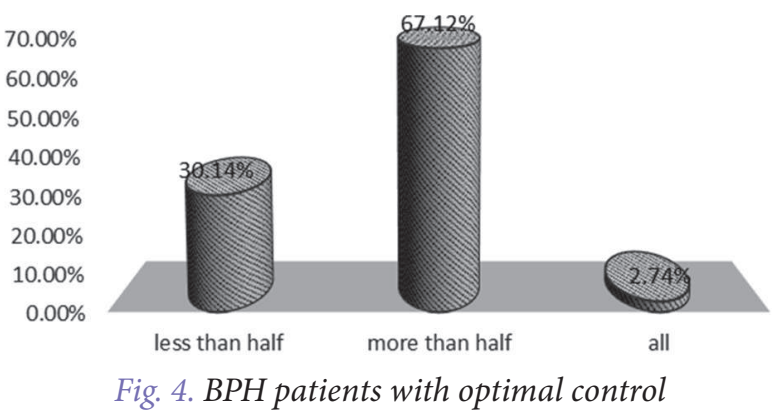

It is obvious from Fig. 5 that $32.88 \%$ of patients in the age group $50-60$ and $63.01 \%$ of those aged 60 70 have complaints related to $\mathrm{BPH}$. The age at which Bulgarian patients visit an urologist coincides with the records regarding the progress of $\mathrm{BPH}$ symptoms.

After the initial detecting of $\mathrm{BPH}$ more than half of the specialists start treatment with alpha-

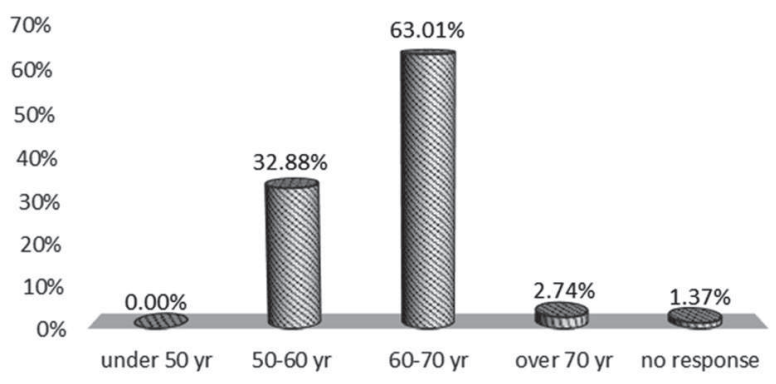

Fig. 5. Age range of patients with $B P H$ blockers $(79.45 \%)$. A combination therapy is preferred by $8.22 \%$. Identical scores are observed in Watchful Waiting and No Response and only 1.37\% of the respondents think that patients should undergo surgical intervention. No one of the interviewed physicians prescribe phytotherapy products, which is a fact worth of note (Fig. 6).

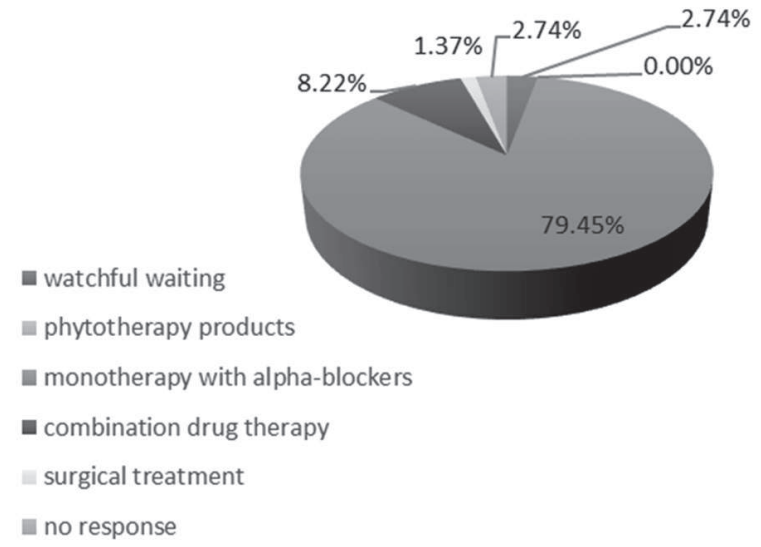

Fig. 6. Approaches after BPH is initially detected

A significant majority of the respondents $(89.78 \%)$ prefer original medicines for the treatment of $\mathrm{BPH}$. A minor score of $5.22 \%$ prescribe generic medicines (Fig 7).

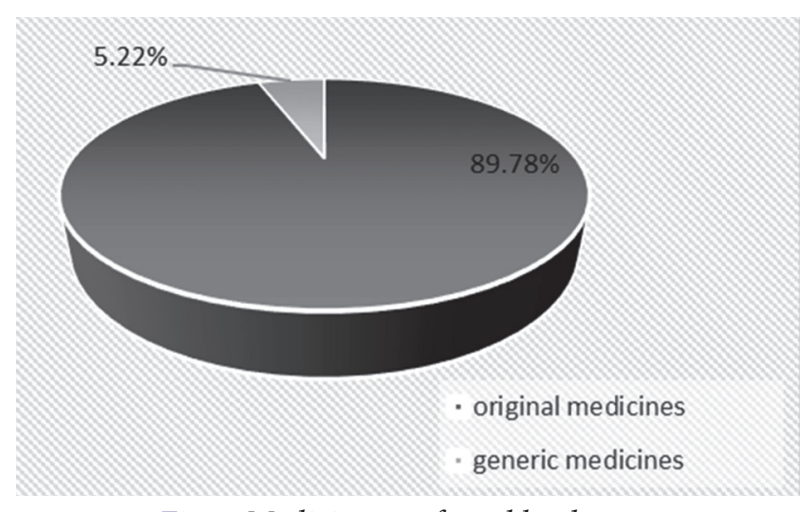

Fig. 7. Medicines preferred by doctors

According to more than half of the interviewed (87.27\%), the original medicines are most effective (Fig. 8).

As a first choice $68.49 \%$ of the medical doctors prefer original Tamsulosin; $19.51 \%$ report about the use of a combination of medicines and $12 \%$ prescribe a generic Tamsulosin (Fig. 9). 


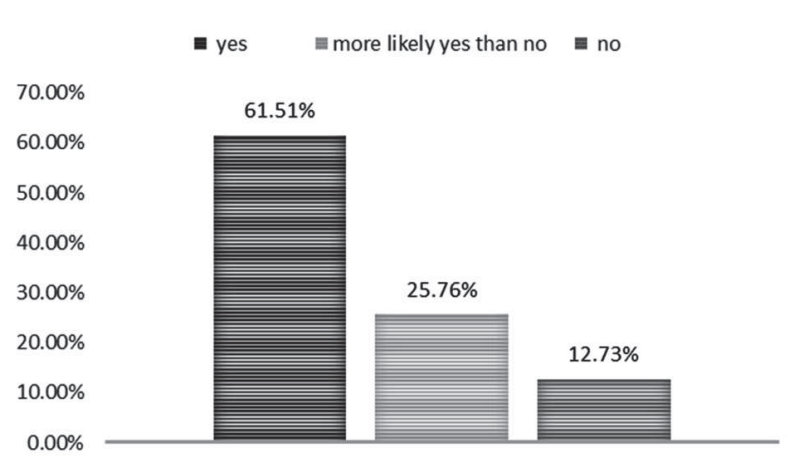

Fig. 8. Effectiveness of original medicines

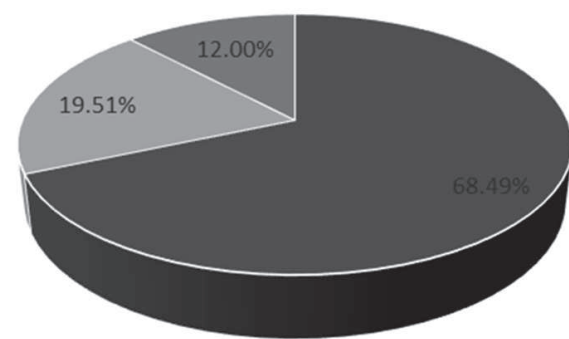

- Original Tamsulosin = Combination Medicine " Generic Tamsulosin

Fig. 9. First medication choice for treatment of $B P H$

The attitude of the urologists is towards treatment of BPH with original Tamsulosin. This could be due to the fact that the medicine concerned is the first original medicine on the Bulgarian market and doctors, apart from having significant experience in the treatment of $\mathrm{BPH}$, have also built trust in the product.

\section{CONCLUSIONS}

The results from the survey indicate the following:

1. After the initial detection of BPH doctors' attitude is towards treatment with an alpha-blocker;

2. To a large degree urologists are familiar with and prefer the original Tamsulosin;

3. The survey indicates that urologists have developed a positive attitude and experience in the use of original Tamsulosin $0.4 \mathrm{mg}$ for the treatment of $\mathrm{BPH}$.

\section{REFERENCES}

1. Wilt T, N'Dow J. Benign prostate hyperplasia Part 1 - Diagnosis. BMJ. 2008;(336):146-150.

2. Guidelines on the Management of Male Lower Urinary Tract Symptoms (LUTS), incl. Benign Prostatic Obstruction (BPO), 2012.

3. Lowe F. Summary of clinical experiences with Tamsulosin for the treatment of benign prostatic hyperplasia. Rev Urol. 2005;7(Suppl 4):S13-S21. 